\title{
A New Method to Characterize Non-oxide Thin Film Uniformity at Device Level using Electron Energy Loss Spectroscopy
}

\author{
Zhi-Peng Li, Lifan Chen, Jianxin Fang, Haifeng Wang, Liang Hong, Xin Jiang, and Yuankai Zheng \\ Western Digital Corporation, Magnetic Heads Operations, 44100 Osgood Road, Fremont, California, \\ USA
}

High quality thin films become critically important as the dimensions in hard disk magnetic recording heads and media continue to shrink down to nanometer/angstrom. It is increasingly challenging to characterize film properties at the device level using conventional methodology developed for large area full films, such as x-ray diffraction, during fabrication process due to limited spatial resolution and sensitivity. Transmission electron microscopy (TEM) with electron energy loss spectroscopy (EELS) has the unique advantage in providing angstrom level spatial resolution in location specific analysis. EELS technique has been well known for characterizing chemical bonding and valence state of transition metal oxide materials by studying the white line ratio and extended energy loss fine structure (EXELFS).

This paper describes an empirical observation of crystalline uniformity through the correlation of EELS white line ratio and EXELFS. This approach demonstrates new EELS application to determine nonoxide thin film crystalline uniformity in magnetic reader structures. Iridium Manganese ( $\operatorname{IrMn}$ ) is a typical antiferromagnetic film used as reader element of the recording head and its crystalline orientation and texture have significant impact on the magnetic performance. Fig. 1 shows the STEM images of two types of IrMn films in complete magnetic reader structure for comparison. Other films above and below are the result of complete film deposition but not the focus of this study, therefore not labeled. The crystalline differences of the two IrMn films are not easily visible by images only, although nanodiffraction analysis (Fig. 2) clearly illustrates that there are more randomness in grain orientations in IrMn film 1 (Fig. 2a).

TEM cross-sections of these two films are made with almost identical thickness. EELS spectra are collected with same conditions, e.g., beam current, dispersion, probe size, aperture size, pixel time and resolution, in order for the spectroscopic analysis to be comparable. Six different EELS are collected for each film at random locations, as shown in Fig. 3 and two insets. By normalizing all Mn L3 peaks, the large ratio variation in Mn L2 peaks can be easily visualized in IrMn thin film 1 (Fig. 3a, denoted as the arrow). The sharp Mn L2 and L3 peaks are known as the white lines in transition metal EELS spectra, arising from the core electrons excited into well-defined empty states [1]. Therefore, the white lines intensity ratio change can be used as a fingerprint to interpret the local element environment. However, in this study, there is no oxidation occurred in either film growth or sample preparation processes. The large Mn L2/L3 peaks ratio variation hence correlates well to the high degree of random orientation of IrMn grains of thin film 1. Moreover, since EXELFS is originated from those ejected electrons not filling the empty states but escaping outside the atom (different from those electrons of white lines) [2], a large variation in the EXELFS in IrMn thin film 1 (Fig. 3a, denoted as the dashed ellipse) is expected. The smaller EXELFS variation in Fig. 3 b can be explained by more uniform grain orientations in IrMn thin film 2, consistent with nano-diffraction results. This new approach has been applied to many IrMn systems at various different times and all shows consistent results. We believe this new methodology has 
potential to be an effective alternative to nano-diffraction, and its feasibility of applying to other materials and improved quantification are promising.

References:

[1] D B Williams and C B Carter, "Transmission Electron Microscopy", $2^{\text {nd }}$ Edition (Springer, New York) p.744.

[2] R F Egerton, "Electron Energy-Loss Spectroscopy in the Electron Microscope", 2 nd Edition (Plenum Press, New York and London) p. 238.

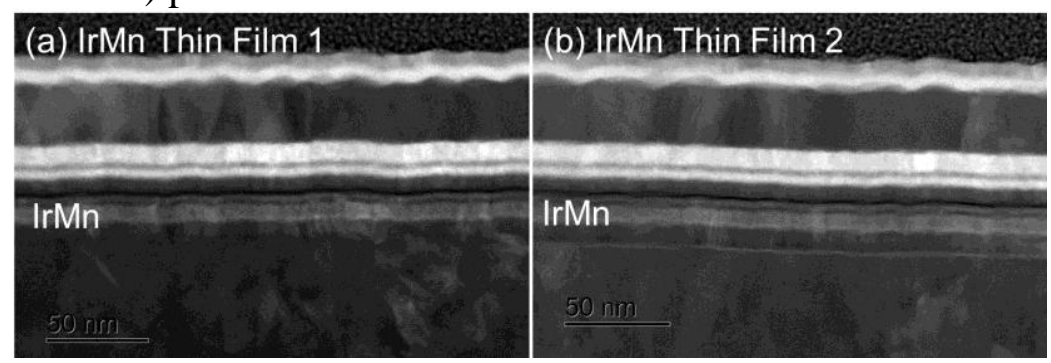

Figure 1. STEM dark field images of two magnetic thin film stacks.

(a) IrMn thin film 1

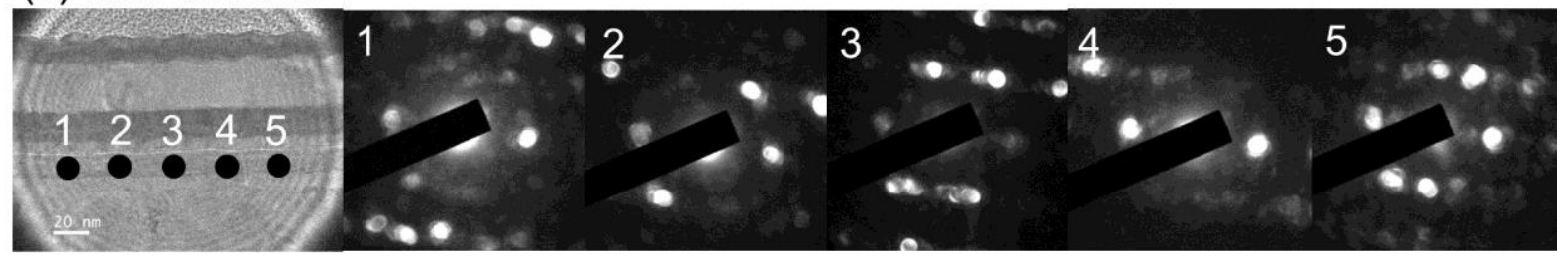

(b) IrMn thin film 2

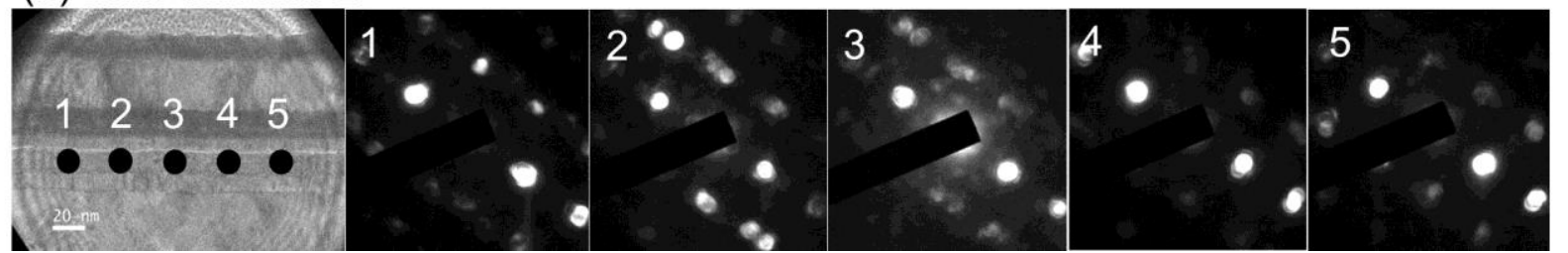

Figure 2. Nano-diffraction patterns of (a) IrMn thin film 1 showing more randomness, and (b) IrMn thin film 2 showing higher degree of texture.

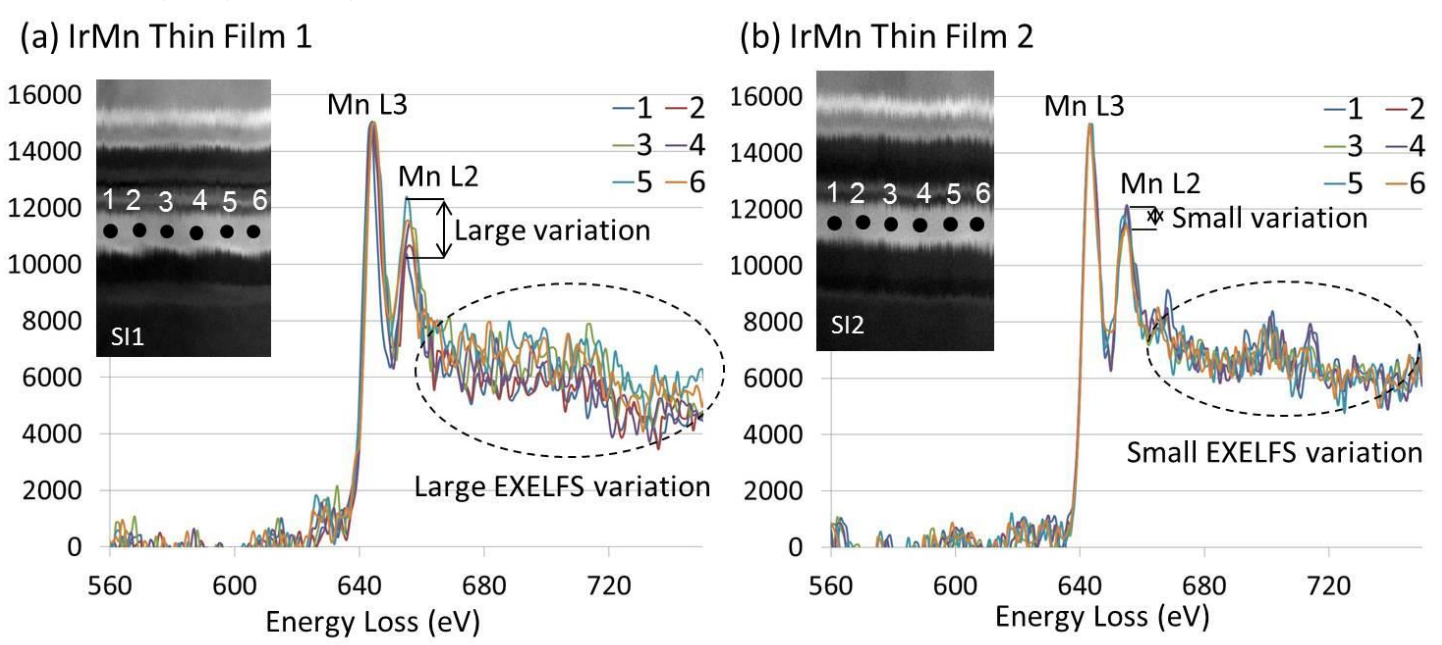

Figure 3. STEM EELS spectra comparison for (a) IrMn thin film 1 and (b) IrMn thin film 2. 